\title{
$7 \mathrm{Ni}-\mathrm{O}$ chemical interaction and the transition temperature of $\mathrm{Ni}$-doped $\mathrm{Bi}_{2} \mathrm{Sr}_{2} \mathrm{Ca}_{1} \mathrm{Cu}_{2} \mathrm{O}_{8}$
}

\author{
P. Alméras, B. Keszei, ${ }^{a)}$ H. Berger, L. Forro, and G. Margaritondo \\ Department of Physics, Ecole Polytechnique Fédérale, CH-1015 Lausanne, Switzerland \\ F. Bussy \\ Institut de Géologie, Université de Lausanne, CH-1015 Lausanne, Switzerland
}

(Received 4 January 1993; accepted for publication 10 May 1993)

\begin{abstract}
We find that even very low $\mathrm{Ni}$ doping levels of high-quality $\mathrm{Bi}_{2} \mathrm{Sr}_{2} \mathrm{Ca}_{1} \mathrm{Cu}_{2} \mathrm{O}_{8}$ single crystals strongly affect the transition temperature $T_{c}$. We also observed that $T_{c}$ is not related to the total $\mathrm{Ni}$ concentration, but only to that of $\mathrm{Ni}$ engaged in NiO-type bonds. By controlling the temperature during crystal growth, one can modify the relative weight of $\mathrm{Ni}$ in NiO-type bonds with respect to other configurations-and therefore $T_{c}$.
\end{abstract}

We present direct experimental evidence that the $\mathrm{Ni}$ doping of $\mathrm{Bi}_{2} \mathrm{Sr}_{2} \mathrm{Ca}_{1} \mathrm{Cu}_{2} \mathrm{O}_{8}$ affects the transition temperature $T_{c}$ through the formation of NiO-like chemical bonds. This clarifies from a chemical point of view a well known doping effect; we find that a photoemission study is required to elucidate this point since the actual dopant concentration dramatically deviates from the nominal one.

The effects of the substitution of copper with other ions in the high-temperature superconductors (HTSC) are being intensively investigated because of their importance in understanding the crucial role of the $\mathrm{Cu}-\mathrm{O}$ planes in superconductivity. ${ }^{1-5}$ It is particularly important to understand the effects of magnetic moments on the superconductivity mechanism. Hence the reason to grow and study systems with magnetic ion substitutions such as $\mathrm{Ni}$. We selected $\mathrm{Bi}_{2} \mathrm{Sr}_{2} \mathrm{Ca}_{1} \mathrm{Cu}_{2} \mathrm{O}_{8}$ rather than $\mathrm{Y}_{1} \mathrm{Ba}_{2} \mathrm{Cu}_{3} \mathrm{O}_{7}$ for our investigations because it has only one (neglecting the superstructure) instead of two equivalent $\mathrm{Cu}$ sites.

Photoemission spectroscopy studies were performed using a SCIENTA ESCA-300 system equipped with a rotating anode (Al $K \alpha$ radiation source), an $x$-ray mono- chromator (composed by seven quartz crystals), and a concentric hemispherical analyzer. With this experimental system we reached an energy resolution of $0.35 \mathrm{eV}$; local analysis over areas as small as a few tens of microns is also possiblc.

The single crystals were cleaved under ultrahigh vacuum $\left(2 \times 10^{-10}\right.$ Torr $)$. The single crystals were grown with the flux method. ${ }^{6}$ We have used different temperature programs, with different stoichiometric compositions, with the objective to increase the Ni concentration. The samples were characterized by $\mathrm{x}$-ray diffraction, fluorescence microprobe (with a CAMEBAX SX-50 instrument), and by resistivity measurements.

Wc have measured large differences between the initial composition of the powder and that of the single crystal (Table I). This was not surprising because the flux can capture some of the elements. After a systematic search, we did not find any correlation between $T_{c}$ and the total concentration of any of the component elements. This was somewhat surprising, since we did find a relation between $T_{c}$ and the growth temperature program: the critical tem-

TABLE I. Characteristics of the samples investigated in the experiments.

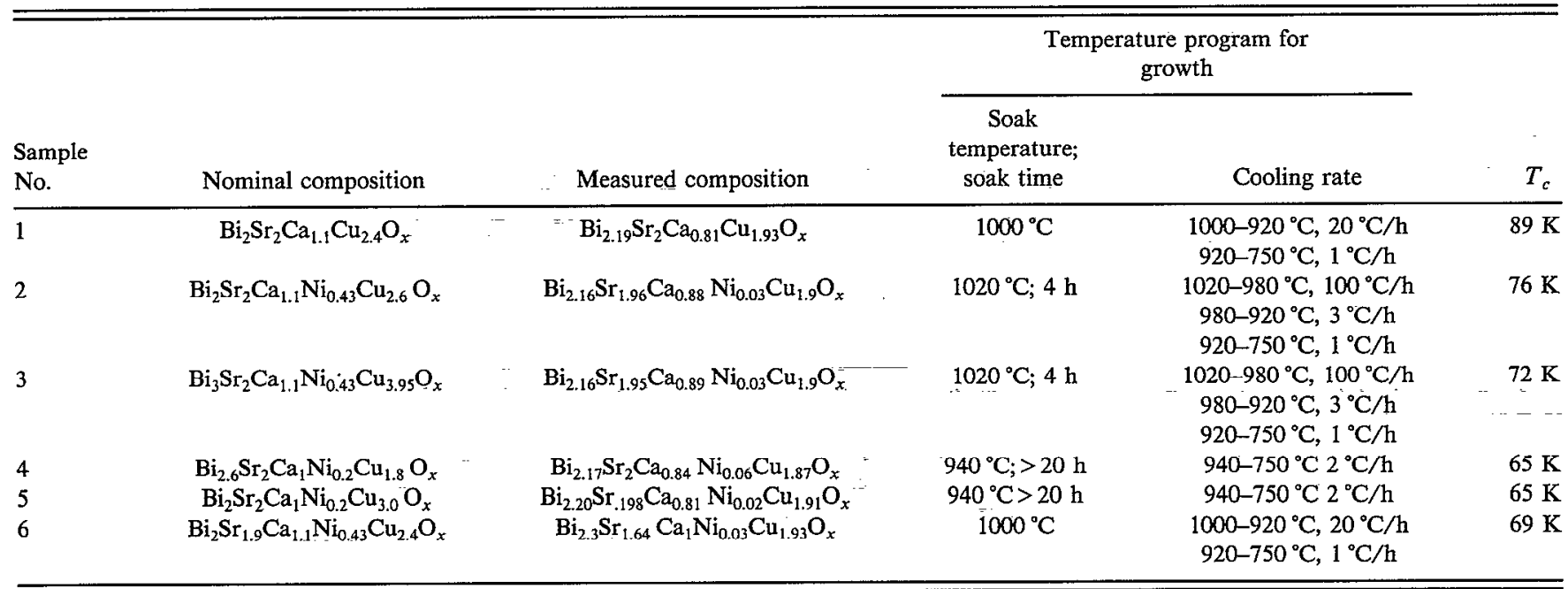

\footnotetext{
a) Present address: Central Research Institute for Physics of the Hungarian Academy of Sciences, H-1525 Budapest, Hungary.
} 


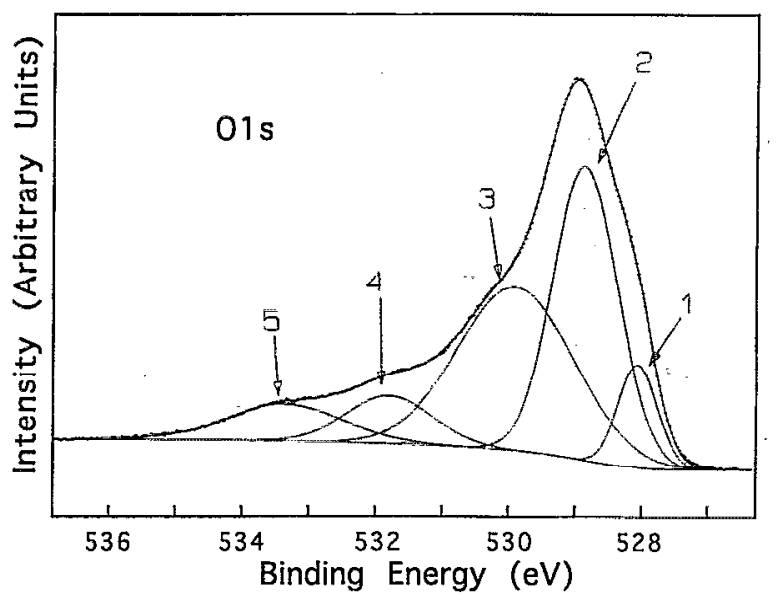

FIG. 1. O $1 s$ of sample 5 showing the different components of peak after deconvolution. Peak 1 is due to $\mathrm{O}$ bond to alkali-earth atoms and $\mathrm{Bi}$ atoms in off-plane sites. Peak 2 is due to the Cu-O planes. Peak 3 is due to oxygen atoms of the $\mathrm{Bi}-\mathrm{O}$ double layers and from NiO-like bonds. Peaks 4 and 5 are due to the sample holder.

perature is reduced with the maximum soak temperature.

Was then this $T_{c}$ decrease related to $\mathrm{Ni}$ or trivially to the quality of the crystals (creation of different phases or microprecipitates)? Our photoemission data favor the first hypothesis. Specifically, we found a relation between $T_{c}$ and the weight of the photoemission $O 1 s$ spectral component related to NiO-like bonds. Consider the $\mathrm{O} 1 s$ spectra of Fig. 1. Oxygen atoms at different cell positions producc ${ }^{7}$ three main spectral components: the $528.1 \mathrm{eV}$ peak is due to $\mathrm{O}$ bound to alkali-earth atoms and $\mathrm{Bi}$ atoms in off-plane sites; the 528.87 and $529.9 \mathrm{eV}$ peaks to the $\mathrm{Cu}-\mathrm{O}$ planes and to the Bi-O double layers.

The relative weights of these components was determined by a least-square fitting. As shown in Fig. 2, the area of peak 3 increases relatively to peaks 1 and 2 , when $T_{c}$ decreases. No other systematic correlation was found, in particular, for the spurious peaks 4 and 5 .

The Ni contribution to peak 3 is related to NiO-like bond spectral features at 529.1 (Ref. 8 and 9) or at 529.6 $\mathrm{eV} .{ }^{10}$ Therefore, we have a relationship between the critical temperature and $\mathrm{Ni}$ in NiO-like bonds. As there is no relation between the total $\mathrm{Ni}$ concentration in the sample and

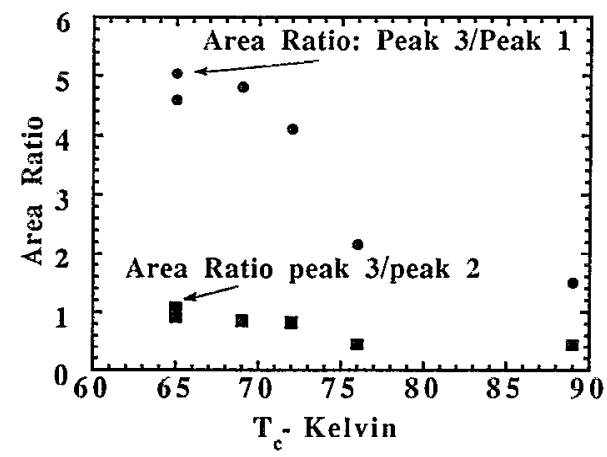

FIG. 2. Area ratios of component 3 is relative to components 1 and 2 of peak $O 1 s$ (see Fig. 1). We can see that the area of the third component increases when the critical temperature decreases.

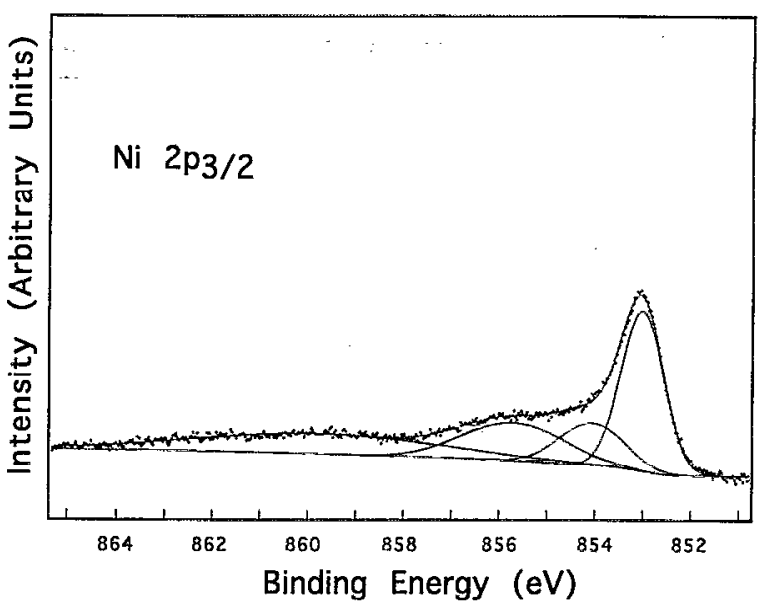

FIG. 3. Ni $2 p_{3 / 2}$ core level spectra of sample 5 .

$T_{c}$, Ni must also exist in other forms (e.g., $\mathrm{Ni}_{2} \mathrm{O}_{3}$ ) or in other planes, without affecting the superconductivity.

The energy overlap of $\mathrm{Ni}-\mathrm{O}$ and $\mathrm{Bi}-\mathrm{O}$ spectral features, and the relatively small amount of $\mathrm{Ni}$, prevent us from identifying which one(s) of the oxygen sites is (are) affected by the formation of Ni-O bonds. In particular, the position in energy of the Ni-O spectral contribution is not sufficient to discriminate between the different sites. Comparative studies with $\mathrm{Y}$ compounds might help clarifying this point.

In order to corroborate the existence of NiO-like bonds, Ni $2 p_{3 / 2}$ spectra like those of Fig. 3 were examined. The main peak is at $852.99 \mathrm{eV}$, the expccted energy for the main NiO feature; note that this peak is above $852.5 \mathrm{eV}$, the binding energy of metallic $\mathrm{Ni}$, and below $855.9 \mathrm{eV}$, the binding energy of $\mathrm{Ni}_{2} \mathrm{O}_{3} \cdot{ }^{8,9}$ The other spectral components due to other configurations of $\mathrm{Ni}$ cannot be easily extracted, since there is a superposition of satellite $\mathrm{NiO}$ features with the main peak of $\mathrm{Ni}_{2} \mathrm{O}_{3}$ at $855.9 \mathrm{eV}$. However, the presence of an $\mathrm{Ni}_{2} \mathrm{O}_{3}$ contribution is clear, since the ratio of the 855.9 spectral component to the main peak at $852.99 \mathrm{eV}$ is not constant from sample to sample.

We have also studied the core levels for other elements (Bi, Sr, Ca), in order to search for possible changes with the Ni concentration. But we did not observe any chemical shift or change in the line shape of these spectra. Similarly, the $\mathrm{Cu} 2 p$ spectrum does not change for different samples. In particular, the $\mathrm{Cu}^{1+}-\mathrm{Cu}^{2+}$ ratio, ${ }^{7}$ usually related to the critical temperature, does not change from sample to sample. Therefore, the incorporation of $\mathrm{Ni}$ in the copper plane does not produce a large change of the oxidation degree of $\mathrm{Cu}$, since this would correspond to a large change in the concentration of carriers ${ }^{11}$ and in the $\mathrm{Cu}^{1+}-\mathrm{Cu}^{2+}$ ratio.

Nevertheless, low-level $\mathrm{Ni}$ doping has a strikingly strong effect on superconductivity. One could envision a perturbing effect of the $\mathrm{Ni}$ magnetic moment on superconductivity (similar phenomena were found for $\mathrm{V}, \mathrm{Cr}, \mathrm{Fe}$, and Co doping ${ }^{3-5}$ ), rather than a direct impact on the carrier concentration; ${ }^{11,12}$ however, strong effects are also observed with a nonmagnetic dopant like $\mathrm{Zn}{ }^{4}$ so the actual mechanism remains unclear. 
This work was supported by the Fonds National Suisse de la Recherche Scientifique and by the Ecole Polytechnique Fćdérale de Lausanne.

${ }^{1}$ P. Murugakoothan, R. Jayavel, C. R. Rao, C. Subramanian, and P. Ramasamy, Mod. Phys. Lett. B 5, 1989 (1991).

${ }^{2}$ P. Kulkarni, S. K. Kulkarni, A. S. Nigavekar, S. K. Agarwal, V. P. S. Awana, and A. V. Narlikar, Physica C 166, 530 (1990).

${ }^{3}$ B. Lönnberg, T. Lundström, and P. Norling, Physica C 191, 147 (1992).

${ }^{4}$ K. Uchinokura, T. Yabe, S. Takebayashi, M. Hase, and A. Maeda, Physica C 162, 981 (1989).
${ }^{5}$ C. M. Lin and S. T. Lin, J. Phys. Condens. Matter 48, 9713 (1991).

${ }^{6}$ P. D. Han and D. A. Payne, J. Cryst. Growth 104, 201 (1990).

${ }^{7}$ H. M. Meyer, D. M. Hill, I. H. Weaver, D. L. Nelson, and K. C. Goretta, Appl. Phys. Lett. 53, 1004 (1988):

${ }^{8}$ K. S. Kim, W. E. Baitinger, J. W. Amy, and N. Winograd, J. Electron. Spectosc. 5, 351 (1974).

${ }^{9}$ K. S. Kim and R. E, Davis, J. Electron. Spectrosc. 1, 254 (1972).

${ }^{10}$ N. S. McIntyre, T. E. Rummery, M. G. Cook, and D. Owen, Electrochem. Soc. 123, 1164 (1976).

${ }^{11}$ H. Srikanth, A. K. Raychaudhuri, C. R. Venkateswara Rao, P. Ramasamy, H. N. Aiyer, and C. N. R. Rao, Physica C 200, 372 (1992). ${ }^{12}$ D. Mandrus, L. Forro, C. Kendziora, and L. Mihaly, Phys. Rev. B 44, 2418 (1991). 